\title{
The Effect of Angiotensin-Converting Enzyme Inhibi- tion on Regional Blood Flow in Salt-Depleted and Salt-Loaded Normotensive Conscious Rats
}

\author{
Kcisuke Nishiyama, M.D., Tadanao Takeda, M.D., Yuji Nakamura, \\ M.D., Masao Ishir, M.D., Hiroaki Matsuoka, M.D., Atsuo \\ Goto, M.D., Kenjiro Kimura, M.D., Yasunobu \\ Hirata, M.S., and Satoru Murao, M.D.
}

\section{Summary}

The effect of angiotensin-converting enzyme inhibition on regional blood flow was studied in a total of 21 normotensive Wistar rats fed on either low or high salt diet. A new potent angiotensin-converting enzyme inhibitor (CEI), SQ 14,225 was administered intravenously in a dose of $2 \mathrm{mg} / \mathrm{Kg}$ to the conscious animals, and changes in fractional distribution of cardiac output were determined with a microsphere method.

Prior to administration of CEI, there was no significant difference in mean arterial pressure (MAP) or regional blood flow between saltdepleted and salt-loaded rats. With CEI, MAP did not change significantly in either group. Fractional distribution of cardiac output increased to the kidneys $(p<0.002)$, and decreased to the stomach, spleen, and skeletal muscle $(p<0.02, p<0.002$, and $p<0.01$, respectively) in the salt-depleted group, while a pattern of blood flow distribution was not changed in the salt-loaded group.

These results suggest that angiotensin II plays an important role in regulating regional blood flow in salt-depleted conscious animals, but not in salt-loaded ones.

\section{Additional Indexing Words :}

Renin-angiotensin system Organ blood flow SQ 14,225 Microsphere

$7 \mathrm{HE}$ availability of the specific inhibitors of the renin-angiotensin system, competitive analogues of angiotensin II and inhibitors of the angiotensin converting enzyme, has provided valuable new tools to precisely evaluate the contribution of this system to circulatory regulation. ${ }^{1-3)}$

Experimental and clinical studies have demonstrated that pharmacoTokyo.

From the Second Department of Internal Medicine, Faculty of Medicine, University of Tokyo,

This study was supported in part by Shimabara Foundation for the Promotion of Science.

Received for publication October $26,1978$.

Manuscript revised January 29, 1979. 
logical inhibition of the renin-angiotensin system increases renal blood flow in two-kidney renovascular hypertension and in some forms of edematous diseases, suggesting the contribution of endogenous angiotensin II in regulating renal circulation under these conditions. ${ }^{4), 5)}$ Recently, a number of studies have shown that angiotensin II may contribute to the control of regional blood flows in other organs as well. ${ }^{6), 7)}$

In normal physiological states, however, it is debatable whether or not the renin-angiotensin system plays any role in the circulatory mechanism, as the administration of the inhibitors of this system does not cause hemodynamic changes unless the subject has been sodium-depleted.8),9) Moreover, recent evidence has shown that the vasculature of different organs displays varying degrees of sensitivity to the action of angiotensin II and other vasoactive agents. ${ }^{10}$ )

Therefore, in an effort to determine whether the renin-angiotensin system contributes to the regulation of regional circulation, and, if any, how the contribution differs under different sodium balances, the effect of the inhibition of the angiotensin-converting enzyme on blood flow distribution was studied in normal Wistar rats subjected to low or high salt diet. For this purpose, SQ 14,225, a new orally active and potent inhibitor of the angiotensin-converting enzyme, was used in this study.

\section{Materials And Method}

Six-week-old Wistar male rats were purchased commercially and raised on standard rat chow (Oriental) and tap water in the authors' laboratory animal house until 12 weeks of age. The rats were then divided randomly into 2 groups. The first group was provided a salt free diet (salt $0.05 \%$, Oriental) and tap water, and the second group a high salt diet (salt $0.64 \%$, Oriental) and $1 \%$ salt solution as drinking water. The rats of each group were fed on either the salt free diet or the high salt diet for at least 3 weeks and then used for this experiment. The measurement of blood flow distribution was successfully accomplished in a total of 21 rats, ranging from 14 to 19 weeks of age: 11 on the salt free diet and 10 on the high salt diet. The method was reported in the authors' previous paper. ${ }^{11)}$

In brief, the animals were anesthetized with ether, and indwelling polyethylene catheters were placed in the left ventricle via the right common carotid artery and in the abdominal aorta through the femoral artery. Five hours after complete recovery from the anesthesia, they were transferred to a small cage in order to measure arterial pressure and to inject microspheres without restraint. The femoral catheter was then connected to a pressure transducer for recording arterial pressure curves on a multichannel polygraph. The position of the ventricular catheter tip was confirmed prior to microsphere injection by obtaining ventricular pressure waves and anatomically, at autopsy, at the conclusion of the study.

Radioactive microspheres (3M Co) with a diameter of $15 \mu \mathrm{m}$, labeled with 
either ${ }^{85} \mathrm{Sr}$ or ${ }^{141} \mathrm{Ce}$, wcre sealed in a silastic tubing for counting individual radioactivity. Following ultrasonic agitation, the tubing was connected to the ventricular catheter and one of the microsphere radionuclides then injected with a $5 \%$ dextrose solution ( $0.4 \mathrm{ml}$ in volume) over a $1-$ min period. After the microsphere injection, a dose of $80 \mathrm{ng} / \mathrm{Kg}$ of angiotensin I (Protein Research Institute) and angiotensin II (Hypertensin, Ciba) were injected in a bolus through the left ventricular catheter to determine the pressor response to each agent. Subsequently, $2 \mathrm{mg} / \mathrm{Kg}$ of $\mathrm{SQ}$ 14,225 was injected systemically. Twenty min later, the microspheres, labeled with another nuclide, were injected into the left ventricle. Pressor responses to angiotensin I and angiotensin II were again examined.

At the conclusion of the study, all the animals were killed by exsanguination and the major organs (i.e., heart, kidneys, brain, lungs, stomach, intestine, liver, pancreas, spleen, adrenal glands, testes, quadriceps muscle, and a patch of skin) were removed and weighed. Organ radioactivity was then measured in a gamma well-type scintillation counter using a pulse height analyzer. Appropriate geometric correction was applied for the volume difference of these organ samples. Fractional distribution of cardiac output to each organ (\% $\mathrm{CO})$ was expressed as percent radioactivity of injected dose.

The results are presented as mean $\pm \mathrm{SE}$. All data were analyzed by Student's t-test for paired or group comparison and a $p$ value of less than 0.02 was considered significant.

\section{Results}

Mean arterial pressure, heart rate, and the magnitude of the pressor response to angiotensin I and angiotensin II in the 2 groups were shown before and after the administration of SQ 14, 225 in Table I. During a control period there was no significant difference in any of these parameters between the 2 groups. The administration of SQ 14,225 caused a transient decrease in mean arterial pressure in both groups, but the decreased pressure returned

Table I. Hemodynamic Characteristics of Salt-Depleted and Salt-Loaded Rats Before and After the Administration of SQ 14,225

\begin{tabular}{l|c|c|c}
\hline & Diet & Control & After CEI \\
\hline Mean arterial pressure (mmHg) & D & $111 \pm 4$ & $105 \pm 3$ \\
Heart rate (beat/min) & L & $107 \pm 3$ & $104 \pm 3$ \\
& D & $393 \pm 9$ & $402 \pm 13$ \\
Pressor response to angiotensin I & L & $389 \pm 22$ & $390 \pm 20$ \\
(mmHg) & D & $15 \pm 1$ & $0^{*}$ \\
Pressor response to angiotensin II & L & $17 \pm 1$ & $0 *$ \\
(mmHg) & D & $21 \pm 2$ & $24 \pm 2$ \\
& L. & $23 \pm 2$ & $26 \pm 2$
\end{tabular}

Each of angiotensin I and II was administered in a bolus dose of $80 \mathrm{ng} / \mathrm{Kg} . \quad \mathrm{D}$, salt-depleted rats; $\mathrm{L}$, salt-loaded rats; CEI, SQ 14,225. * $\mathrm{p}<0,001$. 
to the control level in $20 \mathrm{~min}$, and, therefore, the change was not statistically significant in either group. No significant change was also noted in heart rate. After administration of $\mathrm{SQ}$ 14,225, a bolus injection of angiotensin I failed to induce any pressor response in either group, whereas angiotensin II did cause the same amount of pressor response.

Fractional distribution of cardiac output to each organ is presented in Fig. 1. With respect to the skeletal muscle and skin, fractional distribution was expressed in terms of organ weight. In spite of the difference in salt intake, there was no significant difference in the flow fraction to any individual organ between the 2 groups before the administration of the angiotensin converting enzyme inhibitor. However, these 2 rat groups did
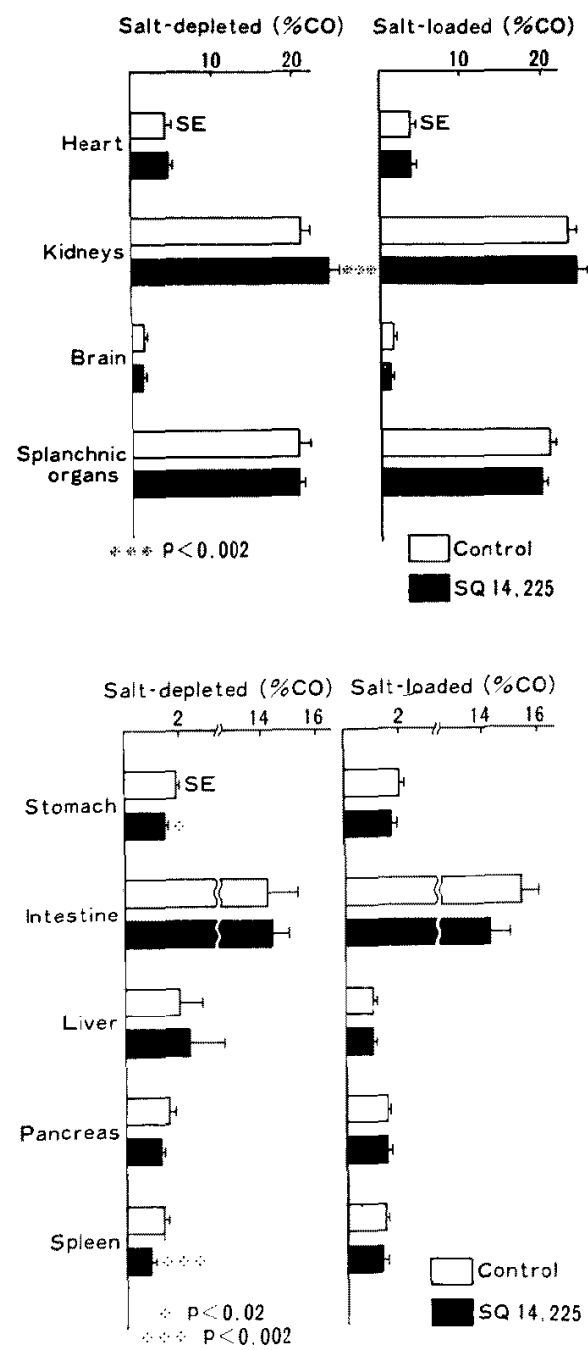

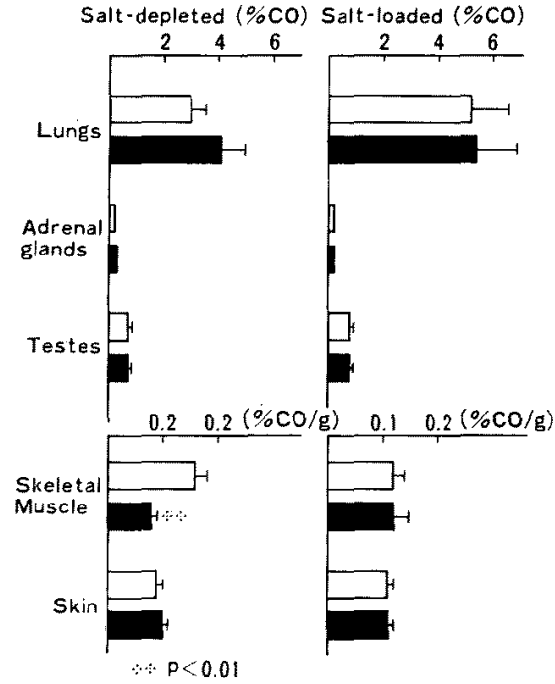

Fig. 1. Fractional distribution of cardiac output to various organs in salt-depleted and salt-loaded rats before and after the administration of $\mathrm{SQ} \mathrm{14,225.} \mathrm{The} \mathrm{flow} \mathrm{fractions} \mathrm{to} \mathrm{the}$ skeletal muscle and skin are shown as percent cardiac output per wet weight. Splanchnic organs include the stomach, intestine, liver, pancreas and spleen. The liver and the lungs refer to the hepatic and bronchial arteries, respectively. $\% \mathrm{CO}$ represents percent cardiac output. The asterisks show a significant difference between the 2 groups. 
show different hemodynamic responses to SQ 14,225. Following the inhibition of the angiotensin-converting enzyme, flow fraction to the kidneys increased from $21 \pm 1.0$ to $24 \pm 0.9 \%(\mathrm{p}<0.002)$, and that to the spleen, stomach, and skeletal muscle decreased significantly $(p<0.002, p<0.02$, and $p<0.01$, respectively) in the salt free diet group, whereas no significant change was observed in the flow fraction to any studied organ in the high salt diet group $(\mathrm{p}<0.02)$.

\section{Discussion}

The effect of SQ 14,225 on regional blood flow was determined with a microsphere method in normal conscious Wistar rats subjected to salt free or high salt diet. The microsphere method used was described in detail and its validity ascertained for measuring fractional distribution of cardiac output to various organs in a previous paper by the authors. ${ }^{11}$

In spite of salt intake difference between 2 groups of rats, neither basal mean arterial pressure nor blood flow distribution to any organ studied was significantly different between them. Following the administration of SQ 14,225 , mean arteial pressure decreased transiently and returned to the basal level 20 min later in both groups. Fractinal distribution of cardiac output increased significantly to the kidncys, and dccreased significantly to the stomach, spleen, and skeletal muscle with the agent in the salt-depleted rats, while no significant alteration was observed in the fractional distribution of cardiac output to any organ studied in the salt-loaded rats.

These data compare favorably with those of previous reports obtained with another converting enzyme inhibitor, SQ 20,881.6),21) Barbourn and Bailie $^{12}$ ) have shown that following the administration of SQ 20,881, blood flow distribution increased to the kidneys and decreased to the spleen in a low dose and to the stomach in a high dose, in normal female rats under pentobarbital anesthesia. Recently, Gavras et al reported the effect of SQ 20,881 on regional blood flow under different sodium balances in normotensive conscious dogs. Their study showed that in the normal sodium diet state, the fractional distribution of cardiac output increased to the adrenal glands and decreased to the lungs and liver after SQ 20,881 administration, while in the sodium-depleted state it increased to the adrenal glands, heart and kidneys, and decreased to the liver and skin.

These studies have suggested in common that the effect of inhibition of the angiotensin-converting enzyme differs on blood flow distribution depending on the sodium balance: a renal fraction of cardiac output increases under sodium depletion, but does not on regular or high sodium diet. 
Recent experimental evidence suggests that the vasculature of different organs may display varying degrees of sensitivity to the action of angiotensin II. The renal vessels exhibit the greatest increase in local resistance after administration of exogenous angiotensin, and the iliac vessels the smallest increase. ${ }^{10)}$ From a theoretical point of view, it is conceivable that inhibition of the renin-angiotensin system increases the blood flow fraction to organs in which vascular beds are most sensitive to angiotensin II, if the vasoconstrictive action of angiotensin II contributes to the regulation of regional blood flow. Hence, the present data, which have shown the increase in renal fraction of cardiac output after SQ 14,225 administration, could be explained exclusively by the effect of inhibition of the renin-angiotensin system on regional blood flow in renin-elevated states.

In the present study, however, other vasoactive effect of SQ 14,225 should be taken into consideration. This compound potentiates bradykinin activity by inhibiting kininase II, as other angiotensin-converting enzyme inhibitors do. ${ }^{13)}$ Accordingly, its potentiation might be responsible for the increase in renal fraction of cardiac output after the administration of $\mathrm{SQ}$ 14,225 in salt-depleted animals. Since changes of bradykinin following alteration of sodium balance generally parallel those of the renin-angiotensin system, augmented and diminished plasma levels of bradykinin are likely present in the salt-depleted and salt-loaded animals, respectively. ${ }^{14)}$ Nevertheless, no significant difference was observed in any organ fraction of cardiac output between the 2 groups before the administration of SQ 14,225. Further potentiation of bradykinin activity following the administration of the compound might have been responsible, in part, for the increase in renal fraction of cardiac output in the salt depleted animals. The possibility, however, seems to be very small, because an increase in flow fraction was not observed to any other organs under salt depletion, and also because there was no significant alteration in fractional blood flow under salt loading.

The decrease in fractional flow to the stomach, spleen, and skeletal muscle in the salt-depleted rats can be hardly explained by the pharmacological actions of the angiotensin-converting enzyme inhibitor. It may be a phenomenon induced secondarily by the increase in fractional flow to the kidneys. Furthermore, the phenomenon may be associated with the evidence that the splenic and muscular vessels exhibit greater constriction than others after adrenergic stimulation. ${ }^{15)}$

In conclusion, alteration in salt intake failed to change the fractional distribution of cardiac output to major organs studied in normotensive conscious rats. Following the administration of an angiotensin-converting enzyme inhibitor, $S Q 14,225$, the fractional distribution of cardiac output increases 
significantly to the kidneys, and decreased to the stomach, spleen, and skeletal muscle in the salt-depleted rats, while no significant change occurred in any organ in the salt-loaded rats. These data suggest that angiotensin II plays an important role in the regulation of regional blood flow in the salt-depleted state.

\section{References}

1. Haber E: The role of renin in normal and pathological cardiovascular homeostasis. Circulation 54: 849, 1976

2. Gavras H, Ribeiro AB, Gavras I, Brunner HR: Reciprocal relation between renin dependency and sodium dependency in essential hypertension. New Engl J Med 295: 1278, 1976

3. Brunner HR, Gavras H, Laragh JH: Specific inhibition of the renin-angiotensin system. A key to understanding blood pressure regulation. Prog Cardiovasc Dis 17: 87, 1974

4. Satoh S, Zimmerman BG: Effect of I-Sar, 8-Ala angiotensin II on renal vascular resistance. Am J Physiol 229: 640, 1975

5. Freeman RH, Davis JO, Vitale SJ, Johnson JA: Intrarenal role of angiotensin II. Homeostatic regulation of renal blood flow in the dog. Circulat Res 32: 692, 1973

6. Gavras H, Liang CS, Brunner HR: Redistribution of regional blood flow after inhibition of the angiotensin-converting enzyme. Circulat Res 43 (suppl I): 59, 1978

7. Messerli FH, Genest J, Nowaczynski, W, Kuchel O, Honda M, Latour Y, Dumont G: Splanchnic blood flow in essential hypertension and in hypertensive patients with renal artery stenosis. Circulation 51 : 1114, 1975

8. Sancho J, Re R, Burton J, Barger AG, Haber E: The role of the renin-angiotensinaldosterone system in cardiovascular homeostasis in normal human subjects. Circulation $\mathbf{5 3}$ : 400,1976

9. Hollenberg NH, Williams GH, Taub KJ, Ishikawa I, Brown C, Adams DF: Renal vascular response to interruption of the renin-angiotensin system in normal man. Kidney Int 12: 285, 1977

10. Heyndrickx GR, Baettcher DH, Vatner SF: Effects of angiotensin, vasopressin and methoxamine on cardiac function and blood flow distribution in conscious dogs. Am J Physiol 231 : 1579,1976

11. Nishiyama K, Nishiyama A, Frohlich ED: Regional blood flow in normotensive and spontaneously hypertensive rats. Am J Physiol 230: 691, 1976

12. Barbour JA, Bailie MD: Effect of the synthetic peptide SQ 20881 on distribution of blood flow in the rat. Proc Exp Biol Med 143: 400, 1973

13. Ondetti MA, Rubin B, Cushman DW: Design of specific inhibitors of angiotensin-converting enzyme: new class of orally active antihypertensive agents. Science 196: 441, 1977

14. Wong PY, Talamo RC, Williams GH, Colman KW: Response of the kallikrein-kinin and renin-angiotensin system in saline infusion and upright posture. J Clin Invest 55: 691, 1975

15. Mark AL, Abboud JM, Schmid PG, Heistad DD, Mayer HE: Differences in direct effects of adrenergic stimuli on coronary, cutaneous and muscular vessels. J Clin Invest 51: 279, 1972 